The International Journal of Engineering and Science (IJES)

|| Volume || 6 || Issue || 4 || Pages || PP 74-79 || 2017 ||

ISSN (e): 2319 - 1813 ISSN (p): $2319-1805$

\title{
The State of Atmosphere Stability and Instability Effects on Air Quality
}

\author{
Osama M. Almethen ${ }^{1}$, Zayed S. Aldaithan ${ }^{2}$ \\ ${ }^{I}$ Navigation Department, Higher Institute of Telecommunication and Navigation, State of Kuwait \\ ${ }^{2}$ Navigation Department, Higher Institute of Telecommunication and Navigation, State of Kuwait
}

\begin{abstract}
The ascending air parcel will expand, and it will experience not only lowering of its pressure and density, but also of its temperature. All three state variables: pressure, density, and temperature will remain in balance as described by the gas law. By combining the equation of state, the first law of thermodynamics, and the hydrostatic equation, the rate of adiabatic temperature change in an ascending air parcel can be found. Atmospheric lapse is a measure of how much air decreases in temperature as it rises through the atmosphere. There are three types of lapse rates: dry-adiabatic, environmental lapse rates, and saturated or moist-adiabatic. The stability of air parcel under vertical displacement is determined by the comparing its laps rate with the environmental laps rate. There are three basic categories in which the atmosphere or a layer in the atmosphere can be classified in terms of stability. These categories are stable, unstable, and neutral. Atmospheric stability is also an extremely important issue in reference to air quality. We can tell how pollutant emissions are likely to disperse and what the likely ground level concentration patterns will be if we know how stable (or unstable) the atmosphere is at a given time. Inversions are associated with minimal air mixing, or more stable atmospheric conditions, and thus generate the highest downwind concentrations from an effluent source.
\end{abstract}

Keywords: atmosphere - air parcel - lapse rates - dry-adiabatic - moist-adiabatic - stability - instability Inversions - air quality - equation of state - hydrostatic equation - thermodynamics - environmental pollutants

Date of Submission:10 April 2017

Date of Accepted: 22 April 2017

\section{INTRODUCTION}

The atmosphere is a three dimensional fluid. The air moves horizontally and vertically; creating weather phenomena, and shaping the properties of climate. The radiation from the sun, while interacting with the Earth's surface and the atmosphere, helps determine the vertical profile of atmospheric temperature. The vertical distribution of heat by convection affects the vertical profile of temperature in a volume of gas or liquid. It is this movement in the atmosphere that leads to the formation of clouds, removes pollutants up and away from the Earth's surface, and helps mix the atmosphere and determine the vertical profile of temperature.

We shall understand the adiabatic processes. As air ascending or descending vertically it cools or heats. These changes in temperature with changes in altitude are called adiabatic processes and they determine the state of the atmosphere, or the stability or instability of the atmosphere. But at first we should be familiar with few concepts of fundamental physical meteorology such as temperature, pressure, density, and how they are governed by the "ideal gas laws"

Towered the end we shall discover how these processes are affecting the air quality of the near surface atmosphere. If we know when certain conditions of stability are most likely to occur and what effect they have on the transport, dispersion, and deposition of pollutants, we can more easily determine when and to what extent emissions should be restricted. City planners and developers can take advantage of this knowledge in order to determine appropriate locations for commercial and residential zoning.

\section{MATERIALS AND METHODOLOGY}

To understand the processes of the atmosphere, it is a must to be familiar with the concepts of temperature, pressure, density, gravity, and heat. Most of atmospheric processes are governed by the laws of thermodynamics, equation of state (ideal gas low), and Newton's laws of motion. Understanding these laws helps quantify these processes, and simplifying the complexity of the atmosphere as a system.

In order to explain how the vertical profile of temperature in the atmosphere is structured to determine the atmospheric state, lets look first at Thermodynamic properties of dry air. It is air that contains no water. The state of a parcel of dry air is described by three properties: 
- temperature $\left(\mathrm{T}\right.$, expressed in ${ }^{\circ} \mathrm{K}$, where $273^{\circ} \mathrm{K}=0^{\circ} \mathrm{C}$ )

- pressure (p, force per unit area, expressed in Newton $/ \mathrm{m}^{2}$ )

- density ( $\rho$, the mass of a unit volume, in $\mathrm{Kg} / \mathrm{m}^{3}$ ).

In a gas these properties are related by a relatively simple physical law called the ideal gas law ideal because it is not exact, although quite accurate for most applications in meteorology. This law states that:

$$
\mathbf{p}=\square \mathbf{R} \mathbf{T}
$$

where, $\mathrm{R}$ is a coefficient, called the gas constant. It does not depend on either $\mathrm{p}, \rho$, or $\mathrm{T}$. The gas constant depends only on the composition of gases that make up the air and every gas has its own gas constant. Since this composition for dry air is roughly constant throughout most of the atmosphere, $\mathrm{R}$ of air is constant and equal to 287 Joules/(kg $\left.{ }^{\circ} \mathrm{K}\right)$.

According to (Dutton, 1995) to understand the equation of state or the ideal gas law, assume that we have a fixed mass of air enclosed in a container with rigid walls, hence there is no change in volume. The ideal gas equation states that the increase in pressure is directly proportional to the increase in temperature. Thus, by warming up the container, say by putting it over a flame, the temperature of the air or the kinetic energy of the air molecules will rises and the pressure, which is the force exerted by these molecules on the container walls will increase. The density of the air will remain constant since there are no increases in the amount of gas, in the container; also there is no increase in the volume of the container. The ideal gas law states that the density decrease will be inversely proportional to the increase in temperature. Therefore, by replacing the rigid wall of the container with flexible ones; allowing it to expand freely, and if the pressure inside rises above that on the outside. In that case, if we raise the temperature, the pressure inside will remain constant, and equal to the outside pressure, but the container's volume will increase. This means that the density will decrease because the mass inside does not change. Moreover, if the source of heat been removed from the flexible walled container, and placing it into a chamber where the pressure can be controlled from the outside, by lowering or increasing it. Remember that the pressure on both sides of the flexible container walls will equalize - as the outside pressure drops, the container will expand and the pressure inside will drop by the same amount. Thus, the density of the air parcel in the container will decrease as well, in agreement with the ideal gas law. But there is no observation to change in temperature because the ideal gas law can not show that Therefore, lets consider the first law of thermodynamics; which a physical law that extends the principle of conservation of energy to include the concepts of heat and work. In thermodynamics the simplest form of energy conservation is the balance between internal energy; the kinetic energy of the body's internal molecular motion is directly proportional to its temperature, and the amount of heat added to the body minus the work done by the body on its surroundings. As the air parcel expands in response to the lowering of the outside pressure, the force of its internal pressure is moving the walls of the container outwards. When a force is moving an object over a given distance it does work. Thus the expanding air parcel does work on its surroundings. This work must come at the expense of internal energy, heat is neither added nor taken away from the parcel in this experiment. Thus the molecular motion within the parcel will slow down, and the parcel's temperature will drop.

As a result, the expanding parcel will experience not only lowering of its pressure and density, but also of its temperature. All three state variables: pressure, density, and temperature will remain in balance as described by the ideal gas law. The process described above is called: adiabatic expansion, implying the change in parcel density without the exchange of heat with its surroundings, and its consequential cooling. The opposite will occur when the parcel is compressed. Adiabatic compression leads to warming.

Secondly, lets take a closer look to another important notation which is the Hydrostatic balance. In the vertical direction, gravity is by far the most important external force acting on the atmosphere. It is the reason for the existence of this crucial envelop of gases around the Earth. The atmosphere does not collapse under the downward pull of gravity because of the energy embedded in the movement of the air molecules. This movement creates the force of pressure which counters the gravitational pull on the atmosphere. The balance between the force of pressure and gravity is the hydrostatic balance. Based on (Ray, 1993) observation of the hydrostatic balance, the atmospheric pressure decreases with height due to the weight of the entire atmospheric column above. As we ascend, there is less of an atmosphere above us, assuming a column of gas $\Delta \mathrm{z}$ meters tall suspended somewhere in the atmosphere; where $\Delta$ symbolizes an interval or difference. The pressure acting on its bottom surface is higher than the pressure acting on its top surface. The pressure difference $\Delta p$ exactly balances the weight per unit area of the column. Therefore, this column of air does not "fall" down under the pull of gravity. It can be stated in mathematical terms this balance can be expressed as:

where $\mathrm{g}$ is the acceleration of gravity $=9.8 \mathrm{~m} / \mathrm{s}^{2}$. 
Thirdly, to find out the rate of adiabatic temperature change in an ascending air parcel (Ahrens, 1991) have point out that it can determined by combining the equation of state, the first law of thermodynamics, and the hydrostatic equation.

$$
\square_{\mathrm{d}}=-\square T / \square Z=9.8^{\circ} \mathrm{C} / \mathrm{km}
$$

where $\Gamma_{\mathrm{d}}$ is adiabatic lapse rate, $\Gamma_{\mathrm{d}}$ is defined as the negative of the actual temperature change $(\Delta \mathrm{T})$, so that $\Gamma_{\mathrm{d}}$ is the amount of cooling that the rising parcel experiences. Sinking air will warm at the same rate as it is being compressed by the increasing pressure.

Since such adiabatic processes occur in the atmosphere, atmospheric lapse rates are often referred to in coincidence with it. The lapse rate is a measure of how much air decreases in temperature as it rises through the atmosphere.

There are three types of lapse rates: environmental lapse rates, dry-adiabatic, and saturated or moist-adiabatic.

- Environmental Lapse Rate: This is the actual measured decrease in temperature with height above the ground (the rate which is actually occurring, not a theoretical rate). Generally this is about $6.5 \mathrm{C}$ per $1000 \mathrm{~m}$. This rate does vary and depends on local air conditions. It can be influenced by several factors; for example: height where lapse rates depend on ground temperature and are normally less near the ground, second seasonal variation where lapse rates are lower in winter or during a rainy season. Third one is surface features where lapse rates are lower over land than sea. And last air masses; different properties of air masses mean different lapse rates.

- Dry Adiabatic Lapse Rate: As the dray parcel of air rises through the atmosphere the surrounding pressure is less and so the parcel expands. Expanding takes energy and so the parcel cools. The rate, at which the parcel cools, stays constant at $9.8 \mathrm{c}$ per $1000 \mathrm{~m}$. It is only applies when the relative humidity is less than $100 \%$. When the air cools to dew point; which is the temperature at which the air can hold no more water without condensing. Water vapor condenses out leading to complications due to the energy introduced from the latent heat. This then means that the saturated lapse rate is used below this temperature.

- Saturated / Moist Adiabatic Lapse Rate: The saturated lapse rate has to take into account the fact the energy is released when water condenses, called the latent heat. This means that once the air has cooled to the dew point and water has started condensing the air parcel cools more slowly. The average saturated adiabatic lapse rate is about $4.5 \mathrm{C}$ per $1000 \mathrm{~m}$.

\section{1) Classification of atmospheric state:}

\section{RESULTS AND DISCUSSIONS}

To determine the stability or instability of the atmosphere (Takle etal.,1975) have pointed out that a comparison must be done between the temperature of a parcel of air, and the temperature of its surroundings at the same height. If the temperature of the parcel is warmer than its surrounding environment, it is more buoyant than its surroundings and will rise higher. If the temperature of the parcel is cooler than its surroundings, it has negative buoyancy and will sink back. In another word, comparing the lapse rate of a parcel of air to the lapse rate of the surrounding air. Motion in the atmosphere makes these comparisons complicated because motion causes changes in the lapse rates we wish to compare. In order to simplify our discussion, we are going to make a few assumptions:

The atmosphere is in hydrostatic equilibrium

- As a parcel of air rises or sinks, there is no compensating motion in the displaced environmental air. The environment around the parcel is static.

- The rising or sinking parcel is isolated from the environment such that the rising and sinking air and the environmental air do not mix. (Adiabatic).

There are three basic categories in which the atmosphere or a layer

in the atmosphere can be classified in terms of stability. These categories are stable, neutral, and unstable.

- If vertically displaced parcels sink back to their initial elevation after the lifting ceases, the environment is stable. (Fig.1)

- If the environment (the surrounding atmosphere) is such that vertically displaced parcels continue to rise on their own, even when the lifting exerted on them stops, the environment is referred to as unstable. (Fig.2)

- If vertically displaced parcels remain where they are after being lifted, the environment is neutral. (Fig.3) 

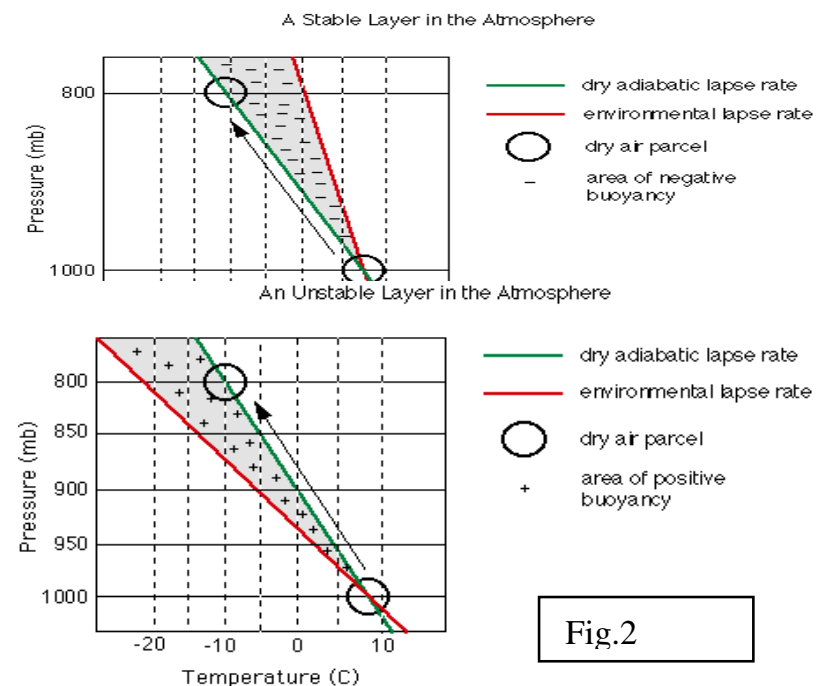

Fig.2

A Neutral Layer in the Atmosphere
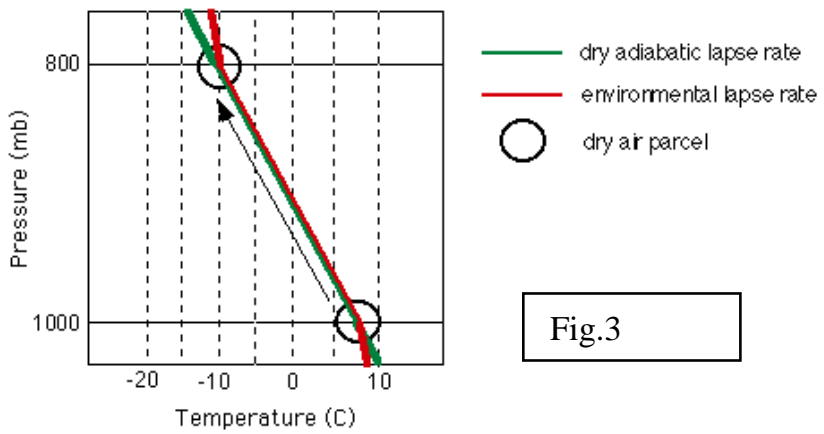

Fig.3

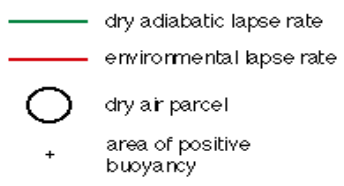

- The Stable Atmosphere is one that is strongly resistant to change. If some external force such as topographic lifting or convergence pushes the air upward, the temperature of the rising air relative to the environment suggests that the air would prefer to go back to its original position. In other words, though a parcel is being forced up, it has negative buoyancy meaning it wants to sink to its original position where it was in equilibrium with the environment. If pushed down, the air has positive buoyancy and wants to rise.

- The Unstable Atmosphere: If a parcel of air is lifted and continues to rise after the lifting force disappears, the atmosphere is unstable. In an unstable layer, the lapse rate of a rising parcel is less than the lapse rate of the environment. Though the parcel cools as it rises, its temperature remains warmer than the surrounding air during its ascent through an unstable layer. Because the parcel is warmer than the environment, the parcel has positive buoyancy and continues to rise on its own.

- The Neutral Atmosphere: While in a stable atmosphere or layer, the lapse rate of a rising air parcel is greater than the lapse rate of the environment. In a neutral atmosphere, the two lapse rates are equal. If a parcel of air is lifted through a neutral layer, the temperature and pressure of the parcel will be identical to the temperature and pressure of the surrounding air at every height and is always in equilibrium with the environment. Thus, the parcel is not buoyant. If the force producing the motion ceases, the parcel will neither continue to rise nor begin to sink, rather, the motion of a parcel will also cease. There could be at least two reasons for the initial displacement of an air parcel:

- An initial warming, through contact with the ground, raises the parcel's temperature, lowers its density, and makes it initially lighter than its environment.

- A vertical push due to an air current hitting an obstacle such as a mountain.

\section{2) The effects of Atmospheric Stability on Air Quality}

Meteorologists are obviously concerned with the stability of the atmosphere for weather forecasting purposes. Knowing the conditions of stability helps to predict the possible development and potential severity of storms. However, atmospheric stability is also an extremely important issue in reference to air quality. We can tell how pollutant emissions are likely to disperse and what the likely ground level concentration patterns will be if we know how stable (or unstable) the atmosphere is at a given time. In addition predicting the transport and dispersion of pollutants based on the wind direction, together with the stability of the atmosphere, meteorologists can predict when certain conditions are more likely to occur and how long they might persist.

Discharge of pollutants to the air during meteorological conditions conducive to their congestion could be reduced or eliminated for many sources of pollution provided adequate and dependable warning of the conditions were given. When conditions are favorable for rapid dispersion and diffusion of contaminants, higher rates of discharge are usually possible without creating undesirable effects. Occasionally meteorological conditions develop which inhibit dispersion of air-borne wastes for extended periods. Forecasts of the latter conditions, coupled with measurements of air quality, could provide a basis for pollution control. (Hoffmann etal., 2008)

Lets focus on such meteorological conditions (stability and instability) that effects mostly the concentration or dispersion of pollutants near ground level, we start firs by defining temperature Inversion which is usually coincide with stability, then moving along to look at the plumes behavior with existent of low level temperature inversion and how it effects the concentration of pollutants. So, temperature Inversions as the study of the lapse rates has shown, the temperature normally decreases with height, but there are certain conditions when this 
does not occur and the temperature rises with height. This is called a temperature inversion (Seinfeld etal., 1998) Temperature inversions occur when warm air overlies colder air. A temperature inversion occurs when temperature increases with height from the ground and can be caused by radiation cooling at the ground or horizontal movement of an air mass from a warm (ground) surface to a cooler surface (water). Temperature inversion can be divided into:

- Major Temperature Inversions: Temperatures increase with height in two levels of the atmosphere, the stratosphere and the thermosphere. The stratosphere contains 4 the majority of the ozone in the atmosphere. As ozone absorbs ultraviolet light from the solar radiation it becomes warm and so the temperatures in this level rise with height. The rise in temperature in the troposphere is due to the absorption of solar radiation by molecular oxygen.

- Minor Temperature Inversions: Temperature inversions can also occur in the troposphere, near the ground and at high levels. High level inversions are found in depressions where the warm air is forced up over the cold air as the warm front is undercut by the cold front. Low level/ ground inversions are normally found in anticyclones conditions when there is a rapid heat loss at night due to radiation or when warm air passes over a cold surface. These

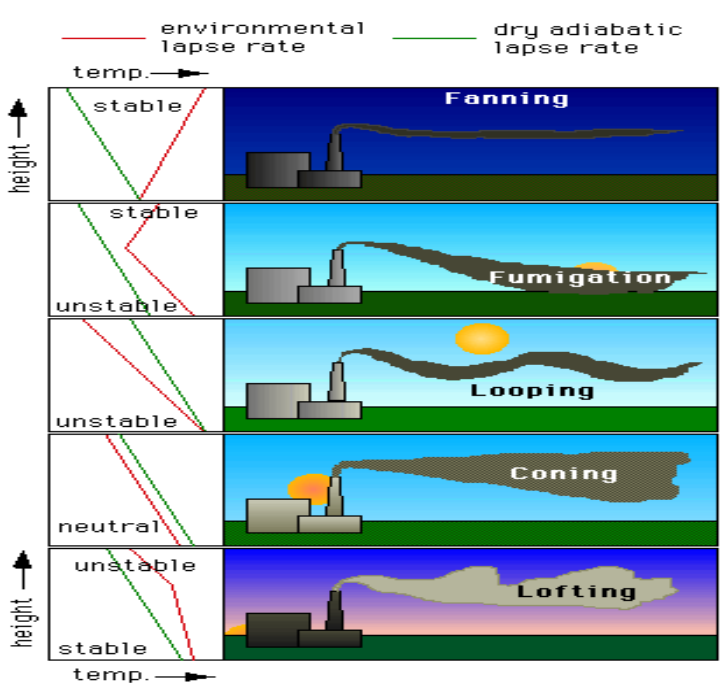
conditions often lead to fog and frost may form in valleys.

\section{3) Low level temperature inversion and concentrations of pollutants:}

According to (Remsberg etal., 1982) inversions are associated with minimal air mixing, or more stable atmospheric conditions, and thus generate the highest downwind concentrations from an effluent source. Meteorological conditions can be described as either stable or unstable, where the stability. Atmospheric stability affects pollution released from ground level and elevated sources differently. In unstable conditions, ground level pollution is readily dispersed thereby reducing ground level concentrations. Elevated emissions, however, such as those released from a chimney, are returned more readily to ground level, leading to higher ground level concentrations. Stable conditions mean less atmospheric mixing and therefore higher concentrations around ground level sources.

The stability of the atmosphere often dictates the behavior of a plume in terms of the height it will raise and to what degree it will mix into the environment (Fisher etal., 2005). For example, a plume released into an unstable atmosphere will display a looping pattern (Fig.4). Looping occurs when updrafts from warming air at the surface carry a segment of the plume upward while compensating downdrafts force the adjacent section downward. Coning occurs when a plume is released in the middle of a neutral layer. In addition to early evening, coning is common at night with the presence of strong winds. A stable atmosphere, commonly marked by an inversion on clear nights, yields a fanning pattern. A plume released into a stable atmosphere will not rise or mix unless it encounters turbulence. A fanning plume will often extend long distances down wind from the source. Looping, coning, and fanning are characteristic of the more persistent conditions of stability, and for this reason, are observed for longer durations over a 24 - hour day. Fumigation and lofting, however, frequently characterize the transition periods between day and night and seldom last for more than a couple of hours. Fumigation occurs in the morning hours as the nighttime inversion gradually disappears due to surface heating. As the surface heats, the air just above it warms. An unstable layer builds from the surface upward but remains capped by the inversion above. A plume released beneath the inversion is trapped near the surface until the inversion eventually disappears and is replaced by an unstable layer. Conversely, lofting occurs in the evening as soon as surface heating ceases and radiation cooling begins.

\section{CONCLUSION}

Low level temperature inversion plays a major role in increasing the concentration of pollutants near ground level. Atmospheric state can be determined by comparing the laps rate of rising air parcel. with its surroundings. In a stable atmosphere, a lifted parcel of air will be colder and heavier than its surrounding then it will sink back, on the other hand in unstable atmosphere, a lifted parcel of air will be warmer than its surrounding and lighter, then it will continue to rise upward away from its original position. It is essential to understand the physics of the atmosphere and how pressure, temperature, and density related mathematically to observe and understand their behavior. There are so many processes in the atmosphere that control or causing different weather 
phenomena, that shape Earth's climate. The most important of them all is the adiabatic expansion. All atmospheric processes are driven by the sun which provides Earth with energy.

\section{REFERENCES}

[1]. John A. Dutton, Dynamics of the Atmospheric Motion, 1995. Dover Publications, INC. New York.

[2]. Peter S. Ray, 1993. Mesoscale Meteorology and Forecasting, American Meteorological Society, Boston.

[3]. C.Donald Ahrens, Meteorology Today: An Introduction to Weather, Climate , and the Environment, 1991. West publishing Company, St.Paul.

[4]. E.S Takle, R.H. Shaw and H.C. Vaughan, 1975. Low-Level stability and pollutant-trapping potential for a rural area, Journal of applied meteorology, Vol. 15, American Meteorological Society, Boston.

[5]. B. Fritz, W. Hoffmann, Y. Lan, S. Thomson, Y. Huang. Low-Level Atmospheric Temperature Inversions: Characteristics and Impacts on Aerial Applications. Agricultural Engineering International: the CIGR journal. Vol. X, May 2008.

[6]. Ellis E. Remsberg and Gerard E. Woodbury 1982, Stability of the surface layer and its relaation to the dispersion of primary pollutant in st. louis. Journal of climate and applied meteorology, Vol.22, American Meteorological Society, Boston.

[7]. Fisher, J. Kukkonen, M. Piringer, M. W. Rotach, and M. Schatzmann, 2005. Meteorology applied to urban air pollution problems. Jornal of Atmospheic Chemistry and physics discussion Vol.5 